\title{
Effect of Intravitreal Bevacizumab with or without Macular Photocoagulation for Diabetic Macular Edema: A Meta-Analysis
}

\author{
Kang Xiao (D) - Shi-Jia Weng · Shen-Zhi Liang · Jiong Wang • \\ Cheng Qian · Guang-Ming Wan
}

Received: August 22, 2018 / Published online: November 2, 2018

(C) The Author(s) 2018

\section{ABSTRACT}

Introduction: In this meta-analysis, we aimed to assess the possible benefits of macular photocoagulation (MPC) as an additional treatment with intravitreal bevacizumab (IVB) in patients with diabetic macular edema.

Methods: The studies were identified from three databases: PubMed, Web of Science, and the Cochrane Library. The main outcome measures included change in best-corrected visual acuity (BCVA), differences in central macular thickness (CMT), and adverse events within the follow-up period. The results were pooled using weight mean difference with their corresponding 95\% confidence intervals. A fixed or random effects model was employed, depending on the heterogeneity of the inclusion trials.

Results: Finally, three randomized controlled trial and two high-quality retrospective studies were identified and included. Changes in CMT at 1,3 , and 6 months did not vary significantly

Enhanced Digital Features To view enhanced digital features for this article go to https://doi.org/10.6084/ m9.figshare.7223939.

K. Xiao · S.-J. Weng · S.-Z. Liang · J. Wang · C. Qian - G.-M. Wan ( $\triangle)$

Department of Ophthalmology, First Affiliated Hospital of Zhengzhou University, Zhengzhou, Henan, China

e-mail: fccwangm5@zzu.edu.cn between the IVB-alone group and the IVB with MPC group $(P=0.26,0.06$, and 0.65 , respectively). Similarly, changes in BCVA at 1,3 , and 6 months also did not vary significantly between the two groups $(P=0.20,0.91$, and 0.70 , respectively). Whereas substantial heterogeneity was detected in the CMT results among these studies, the sensitivity analyses showed Solaiman's study was probably the source of the heterogeneity. No publication bias was detected by funnel plots in this study.

Conclusion: Results of this meta-analysis showed that the treatments with IVB alone and combined IVB and MPC were similarly effective in improving BCVA and reducing CMT. However, more evidence is needed to evaluate their effects in the long-term periods.

Keywords: Bevacizumab; Diabetic macular edema; Macular photocoagulation; Metaanalysis

\section{INTRODUCTION}

Diabetic macular edema (DME), which is the leading cause of vision loss in patients with diabetes, is a common manifestation of diabetic retinopathy characterized by the exudation and accumulation of extracellular fluid and plasma constituents [1-3].

Macular photocoagulation (MPC) has been recommended to reduce the risk of vision loss 
from DME by the Early Treatment Diabetic Retinopathy Study (ETDRS) [4]. It is considered to result in the proliferation of both the endothelial cells in retinal capillaries and the retinal pigment epithelial cells, thus improving the efficacy of both the inner and outer bloodretina barriers [5]. The ETDRS reported that MPC reduced moderate visual loss by $50 \%$, whereas unsatisfactory outcomes are frequent, and $12 \%$ of treated eyes still lost 15 or more ETDRS letters at the 3-year follow-up interval [4]. Furthermore, it was observed that some patients with DME still had poor visual prognosis despite laser photocoagulation $[6,7]$. Hence, more brisk and effective therapies are encouraged to apply in the cure of DME.

As an angiogenic inducer and a vascular permeability factor [8], the upregulation of vascular endothelial growth factor (VEGF) has been believed to be associated with the breakdown of the blood-retinal barrier and an increase in retinal vessel permeability, resulting in macular edema in diabetic eyes $[9,10]$. Hence, anti-VEGF therapy is expected to show an effective reduction in DME. Bevacizumab is a full-length, recombinant, humanized, monoclonal antibody that blocks all forms of VEGF, and many studies have reported that it was effective in reducing DME and improving bestcorrected visual acuity (BCVA) when injected intravitreally $[11,12]$. However, recurrence of macular edema in bevacizumab-injected eyes was observed within a few weeks after the treatment $[13,14]$. The reason was probably due to the limited effective time of bevacizumab because its half-life in the eyes is only 9.8 days [15]. Owing to the temporary effect of intravitreal bevacizumab (IVB), adding another modality is recommended to prolong and stabilize the beneficial effect.

In conclusion, IVB and MPC achieve their effect by different pathways, and the changes induced by MPC in the retinal tissues are permanent, which could probably prolong the effect of IVB injection. Therefore, a combination therapy may yield better visual outcomes or greater decrease in central macular thickness than IVB therapy alone. In this meta-analysis, we evaluate whether intravitreal therapy of bevacizumab has a synergistic effect when combined with MPC and compare this combination with primary IVB therapy alone.

\section{METHODS}

\section{Literature Search}

Three databases were used to conduct a computerized search for relevant available articles published up to August 2018: PubMed, Web of Science, and the Cochrane Library. Search terms used were "bevacizumab," "Avastin," "photocoagulation," and "diabetic macular edema." The language was restricted to English. Furthermore, we reviewed reference lists from retrieved articles to appraise other potential studies.

\section{Inclusion and Exclusion Criteria}

Trials were considered for inclusion if they met the following criteria: (1) randomized controlled trials (RCTs) or high-quality comparative studies, (2) interventional therapies for DME consisting of IVB alone versus IVB combined with MPC (IVB/MPC), and (3) all studies that contained sufficient information about pre- and post-treatment CMT and BCVA in the logarithm of the minimum angle of resolution (logMAR) equivalents measured and recorded as mean \pm standard deviation (SD). The exclusion criteria were the following: (1) studies of macular edema secondary to causes other than diabetic maculopathy and (2) case reports, abstracts, reviews, and reports with incomplete data about macular thickness or best-corrected visual acuity. If different publications from the same study subjects were available, the most recent one was included.

\section{Data Extraction and Quality Assessment}

For each study, the following data were independently extracted by two reviewers: publication metrics (name of the first author, year of publication, location of the study, and study design), population characteristics (number of eyes and mean age), treatment information (dosage of IVB, mean time of IVB during follow- 
up, and type of MPC), duration of follow-up, and treatment outcome in terms of CMT $(\mu \mathrm{m})$ and BCVA (log MAR). In cases of conflicting evaluations, disagreements were resolved through discussion. We used the Cochrane Collaboration's tool for risk of bias to evaluate the quality of RCTs [16]. The following study characteristics were assessed for biases: random sequence generation (selection bias), allocation concealment (selection bias), blinding of participants and personnel (performance bias), blinding of outcome assessment (detection bias), incomplete outcome data (attrition bias), selective reporting (reporting bias), and other factors that contribute to biases. The status of each of these items was listed as a low, high, or unknown risk of bias. Moreover, the modified Newcastle-Ottawa Scale (NOS) was used to perform the quality assessment of retrospective studies [17]. On this scale, a total of four stars were given for patient selection, three for outcome assessment, and two for comparability, and a score of at least 7 stars was considered to be an indication of high quality.

\section{Statistical Analyses}

A meta-analysis on the effect of IVB or IVB/MPC on DME was performed with Cochrane Review Manager (RevMan, version 5.2) software. The treatment effect was estimated by means of the weighted mean difference (WMD) in CMT in $\mu \mathrm{m}$ and BCVA in logMAR equivalents with a 95\% confidence interval $(\mathrm{CI})$, and $P<0.05$ was considered statistically significant. The between-study heterogeneity was tested using the chi-square test and $I^{2}$ statistic. When there was no statistical heterogeneity $(P>0.1$, $\left.I^{2}<50 \%\right)$, a fixed effects model was used; otherwise, the random effects model was used $\left(P \leq 0.1, I^{2} \geq 50 \%\right)$. Sensitivity analyses were also conducted, by which the influence of a single study on the pooled effect was examined by removing one study at a time. In addition, we used funnel plots to identify the publication bias.

\section{Compliance with Ethics Guidelines}

This article is based on previously conducted studies and does not contain any studies with human participants or animals performed by any of the authors.

\section{RESULTS}

\section{Literature Search}

The selection process for inclusion of studies in this meta-analysis is summarized in Fig. 1. A total of 297 studies that were potentially relevant were yielded by electronic literature searches, and 31 were excluded because they were duplicate studies. Then, 257 studies were also excluded after skimming through the titles and abstracts. Consequently, nine studies remained for further assessment and a full-text review. Among them, three studies were excluded because they did not meet the inclusion criteria and another was omitted for providing insufficient data. Ultimately, five studies [14, 18-21], including three RCT trials and two retrospective studies, met the inclusion criteria and were included in this meta-analysis.

\section{Characteristics of the Studies and Quality Assessment}

Table 1 shows the detailed characteristics of the five included studies. Briefly, studies were conducted in several countries: Iran, Egypt, Korea, the USA, and Serbia. Of them, three were RCT trials and two were retrospective studies. Sample sizes varied from 43 to 298 subjects, and durations of follow-up varied from 1 to 24 months. In all the studies, the distribution of age, gender, and history of DME did not vary significantly between the IVB and the IVB/MPC groups. The risk-of-bias assessment of the included RCTs is presented in Fig. 2. Faghihi's study performed randomization with the random dot table, whereas the randomization method was unknown in another two studies. Moreover, allocation concealment was not mentioned in any study. Only Jovanović's 


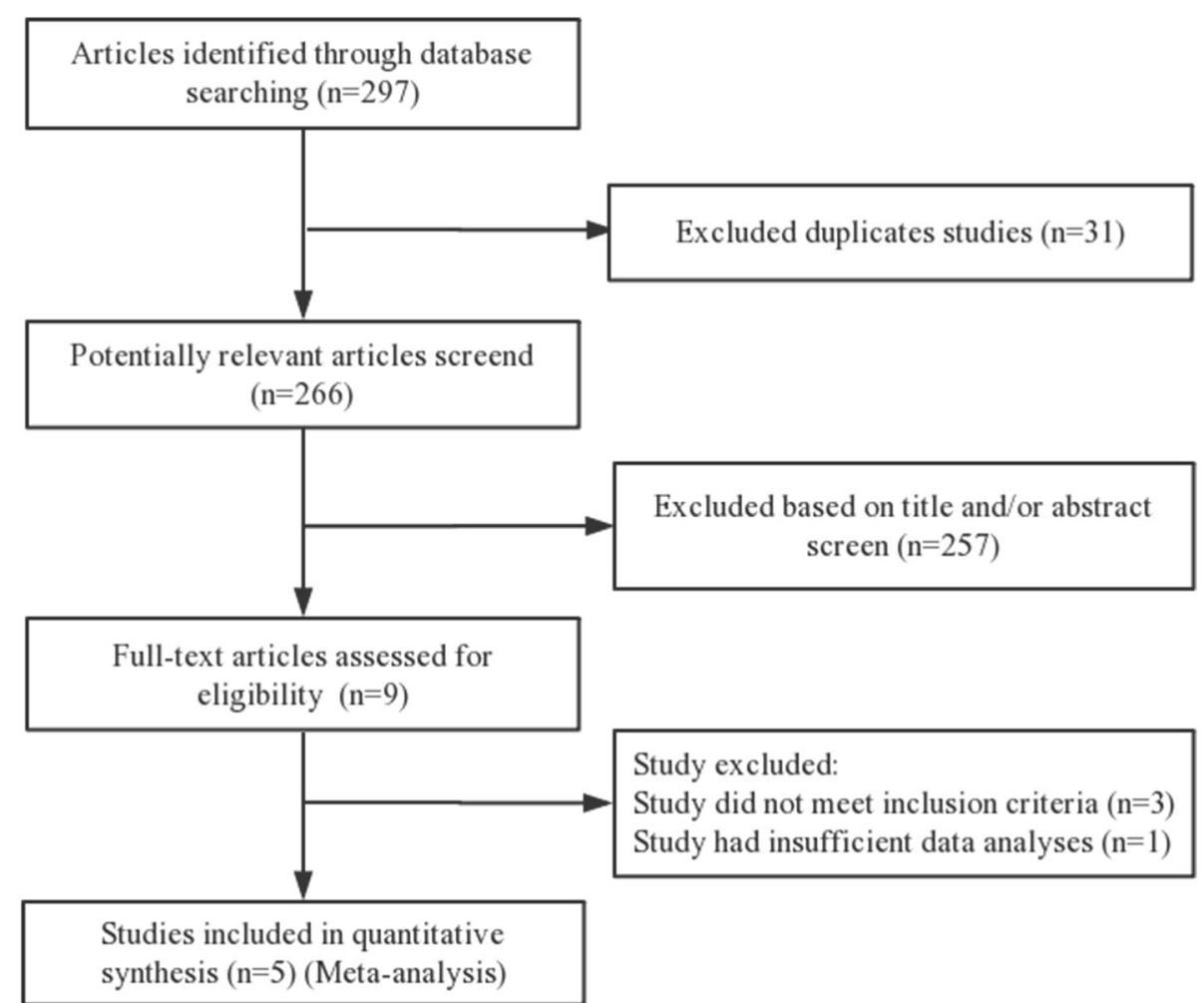

Fig. 1 Flow diagram of study selection

mentioned blinding of patients, and whether another two studies were conducted in a blinded fashion is unknown. There was no loss of follow-up in any of the studies, and all of them were free of reporting or any other bias. The NOS system was used to assess the quality of the retrospective studies. Both studies had three stars for selection (four stars maximum), two stars for comparability (two stars maximum), and three stars for outcome assessment (three stars maximum). Both studies scored more than seven, indicating a low risk of bias.

\section{Central Macular Thickness}

CMT represented the anatomic change of DME levels, and three studies reported CMT at 1 month after the initial treatment (Fig. 3). There was statistical heterogeneity among the studies $\left(P=0.02, I^{2}=75 \%\right)$, and a random effects model was used. Both interventions resulted in decreased CMT, and IVB did not vary significantly compared with IVB/MPC
$(\mathrm{WMD}=-18.82 ; 95 \%$ CI -15.26 to 13.61 ; $P=0.26$ ). Two studies reported data on CMT at 3 months after the initial treatment. Significant heterogeneity was observed $(P=0.0003$, $I^{2}=92 \%$ ), so the random effects model was selected. The changes in CMT did not vary significantly between the IVB and IVB/MPC groups $(\mathrm{WMD}=59.03 ; \quad 95 \% \quad \mathrm{CI}-2.63$ to 120.69 ; $P=0.06)$. Regarding the CMT at 6 months after the initial treatment, it showed statistical heterogeneity $\left(P=0.00001, I^{2}=93 \%\right)$, and the changes in CMT did not vary significantly between IVB and IVB/MPC (WMD = 12.63; 95\% CI -42.38 to $67.63 ; P=0.65)$.

\section{Best-Corrected Visual Acuity}

As a functional outcome measure, BCVA was extremely important to evaluate the treatment efficacy. The result of BCVA was converted to a logarithm of the minimum angle of resolution ( $\log$ MAR) vision and was summarized by means of meta-analysis. Figure 4 shows the effects of 


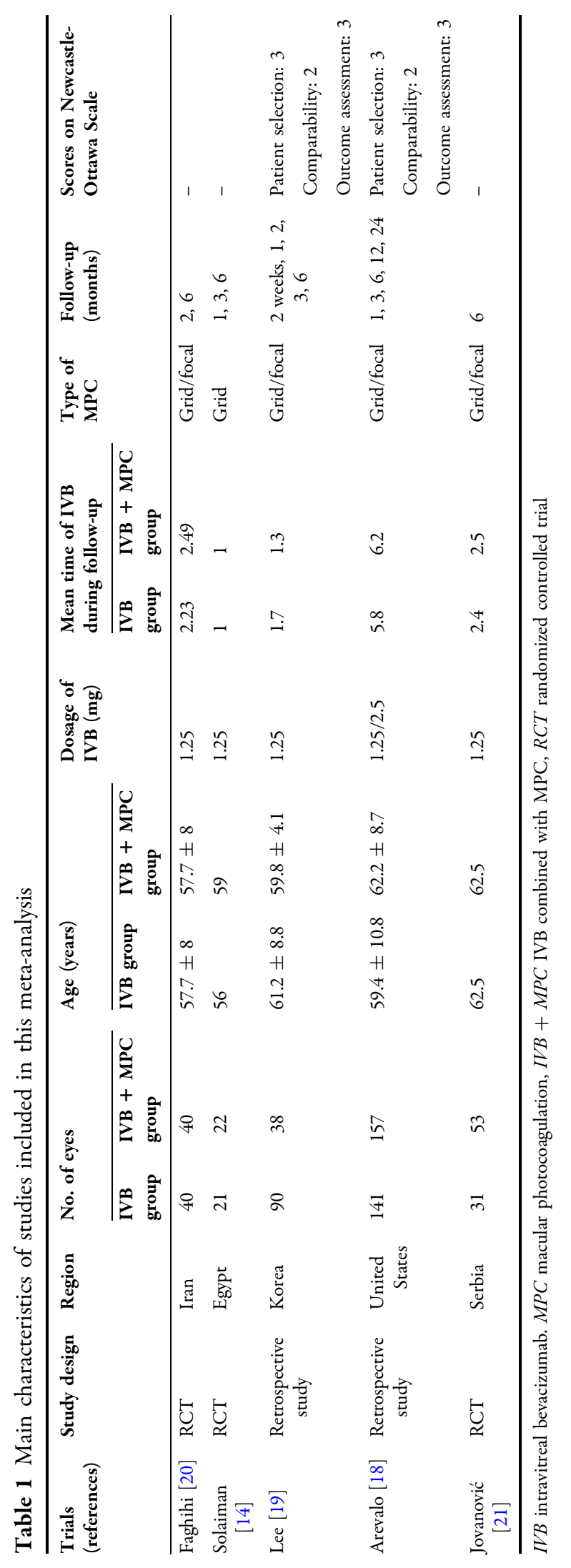




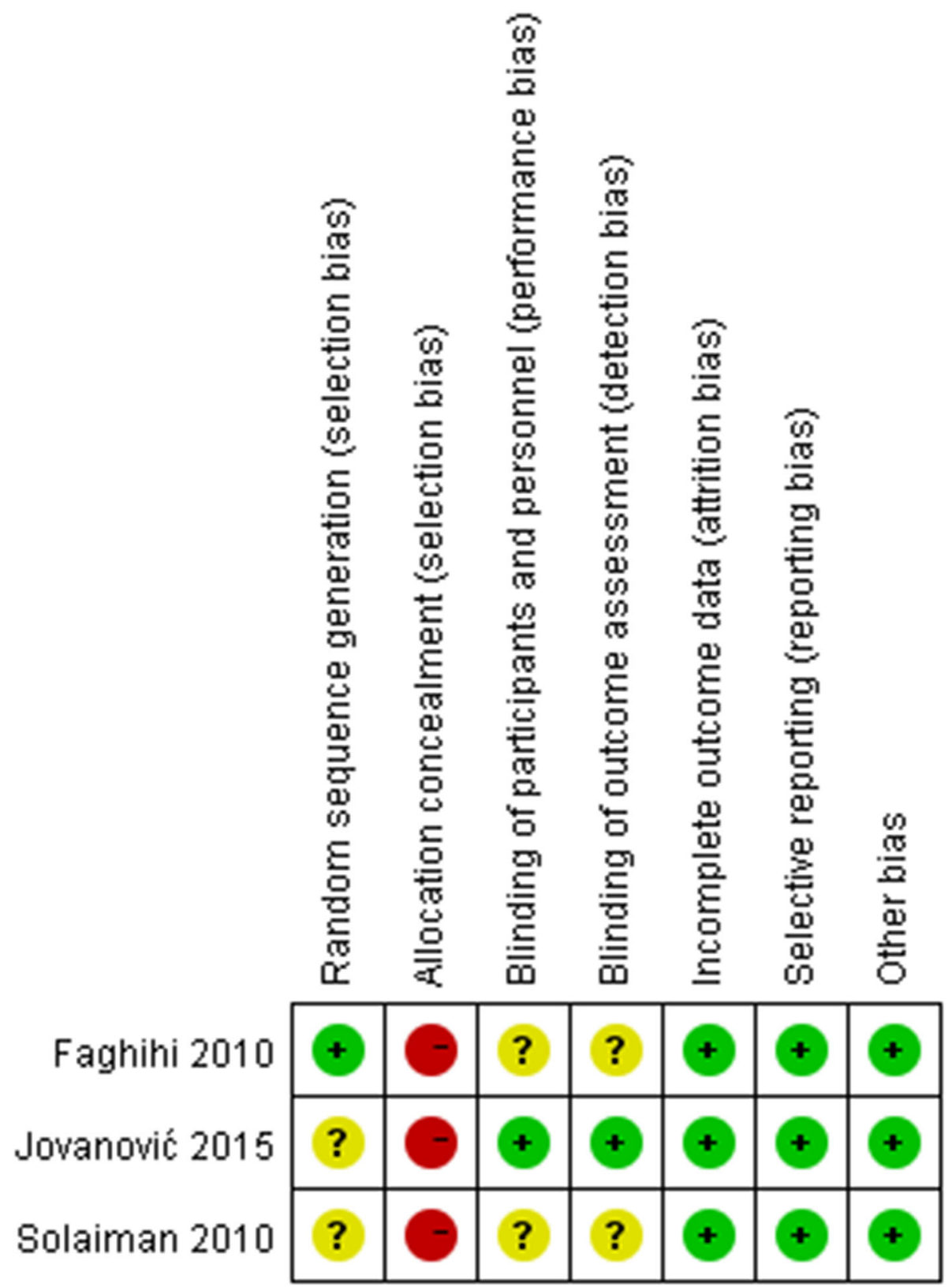

Fig. 2 Assessment of bias risk for all randomized controlled trials (RCTs). Bias risk was classified as low (+), unclear (?), or high (-)

the IVB and the combined therapies on BCVA by creating the forest plot. Three studies showed the BCVA at 1 month after the initial treatment with no statistical heterogeneity among trials $\left(P=0.21, I^{2}=37 \%\right)$. A fixed effects model was used, and the changes in BCVA at 1 month after the initial treatment did not vary significantly between the IVB and combined treatment groups $(\mathrm{WMD}=-0.04 ; 95 \% \mathrm{CI}-0.10$ to 0.02 ; $P=0.20$ ). Three studies reported BCVA at 3 months after the initial treatment and demonstrated no statistical heterogeneity among trials $\left(P=0.64, I^{2}=0 \%\right)$. Again, changes in BCVA did not vary significantly between the IVB and combined treatment groups $(\mathrm{WMD}=-0.00 ; \quad 95 \% \quad \mathrm{CI}-0.06$ to 0.06 ; 

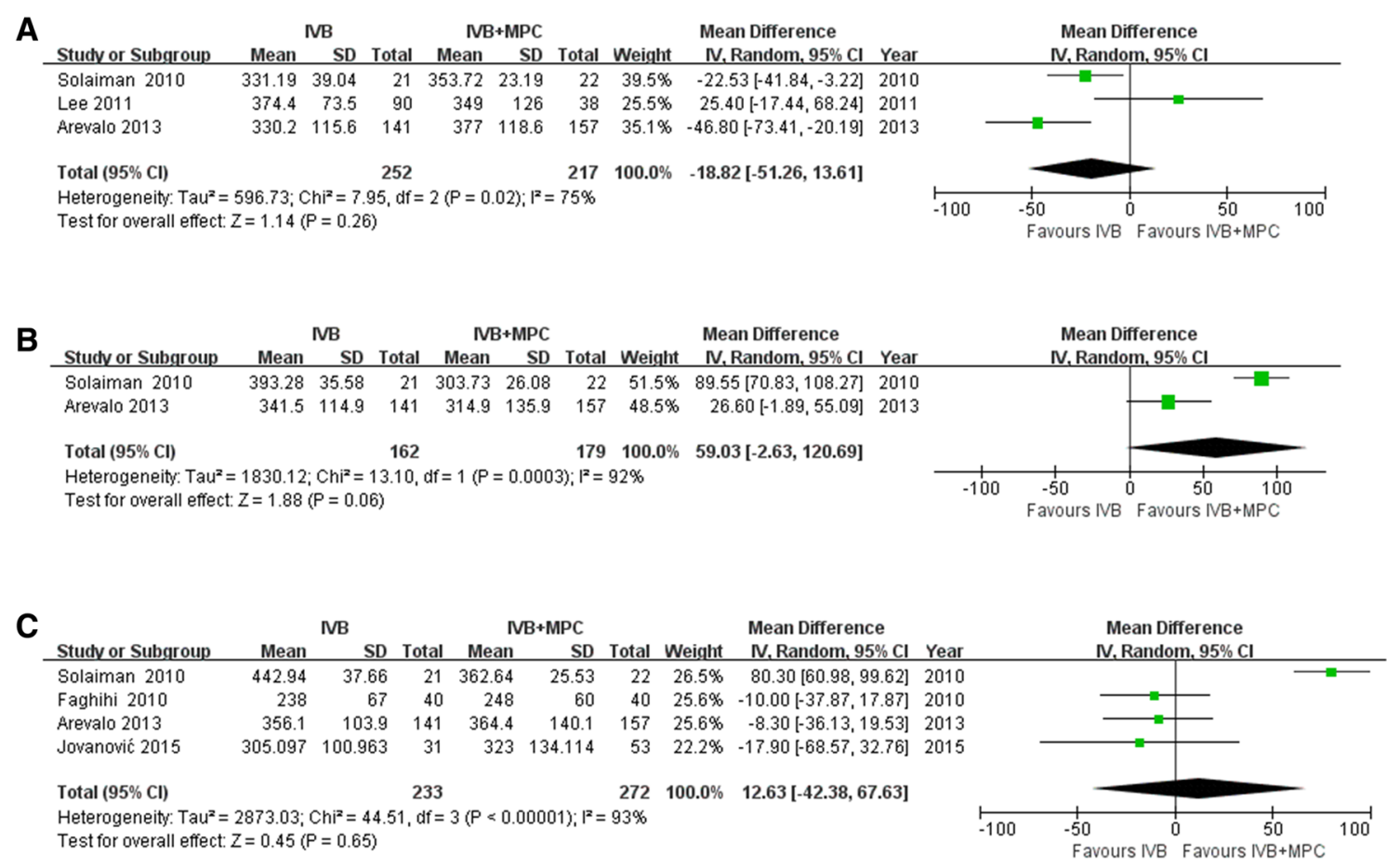

Fig. 3 Forest plots showing the mean differences in central macular thickness (CMT) along with associated 95\% confidence intervals (CIs) comparing IVB with IVB + MPC at 1 (a), 3 (b), and 6 (c) months. Negative values in these plots favor IVB over IVB + MPC; positive

$P=0.91)$. All five studies showed data on BCVA at 6 months after the initial treatment. There was no statistical heterogeneity $(P=0.54$, $I^{2}=0 \%$, and changes in BCVA also did not vary significantly between the IVB and combined treatment groups $(\mathrm{WMD}=-0.01 ; 95 \%$ CI -0.06 to $0.04 ; P=0.70$ ).

\section{Adverse Effects}

All the studies reported the absence of serious complications, such as an increase in intraocular pressure, vitreous hemorrhage, endophthalmitis, cataractous change, or systemic adverse effects in both groups.

\section{Sensitivity Analysis and Publication Bias}

The results of the leave-one-out analysis on the CMT at 6 months showed that all exclusions did not alter the results of the previous analyses, values favor IVB + MPC over IVB. IVB intravitreal bevacizumab, $I V B+M P C$ intravitreal bevacizumab combined with macular photocoagulation

suggesting reliability and stability of the results of this meta-analysis (Table 2). In addition, the sensitivity analysis found that Solaiman's study was the source of the heterogeneity. After excluding Solaiman's study, the pooled WMD was $-10.30(95 \%$ CI $-28.65,8.06)$, with no evidence of heterogeneity $\left(P=0.95, I^{2}=0 \%\right)$. The funnel plot for BCVA at 6 months was revealed. According to the funnel plots, the studies were within the confidence intervals, and the shapes of the funnel plots did not reveal any evidence of obvious asymmetry (Fig. 5).

\section{DISCUSSION}

The present meta-analysis included three RCTs and two retrospective studies to compare the therapeutic effects of IVB with or without MPC for DME. In our analysis of CMT and BCVA at 1, 3 , and 6 months after the initial treatment, no significant difference was found between the 

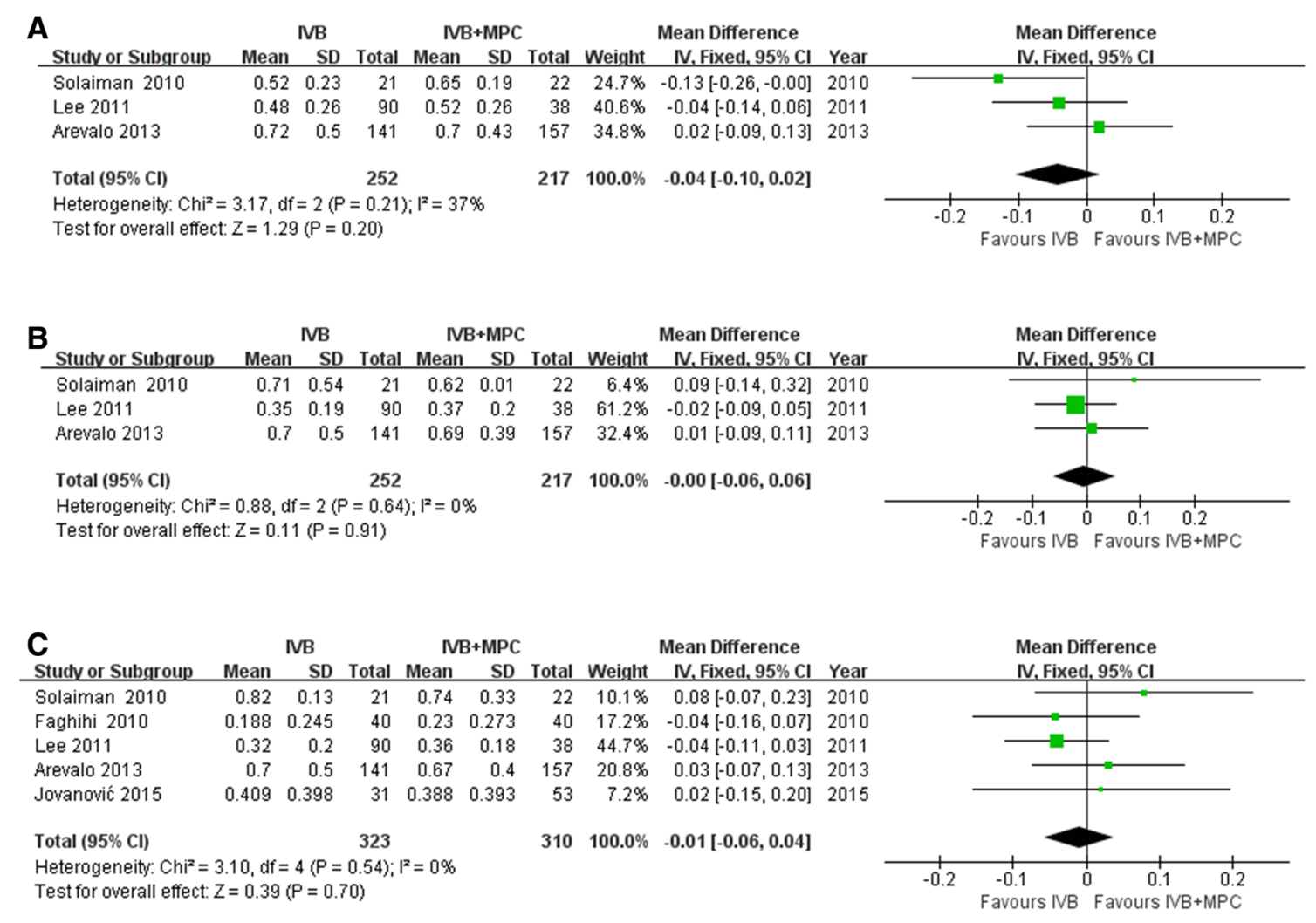

Fig. 4 Forest plots showing the mean differences in bestcorrected visual acuity (BCVA) along with associated $95 \%$ confidence intervals (CIs) comparing IVB with IVB + MPC at 1 (a), 3 (b), and 6 (c) months. Negative values in these plots favor IVB over IVB + MPC; positive values favor IVB + MPC over IVB. IVB intravitreal bevacizumab; $I V B+M P C$ intravitreal bevacizumab combined with macular photocoagulation

Table 2 Outcomes for sensitivity analysis of included studies evaluating CMT at 6 months

\begin{tabular}{|c|c|c|c|c|c|c|c|}
\hline \multirow[t]{2}{*}{ Study excluded } & \multicolumn{2}{|c|}{ Random effects model } & \multicolumn{3}{|c|}{ Test for heterogeneity } & \multicolumn{2}{|c|}{ Test for overall effect } \\
\hline & $\overline{M D}$ & $95 \% \mathrm{CI}$ & $\bar{Q}$ & $I^{2}(\%)$ & $P$ value & $\bar{Z}$ & $P$ value \\
\hline None & 12.63 & $-42.68,67.63$ & 44.51 & 93 & $<0.00001$ & 0.45 & 0.65 \\
\hline Solaiman $[14]$ & -10.30 & $-28.65,8.06$ & 0.11 & 0 & 0.95 & 1.10 & 0.27 \\
\hline Faghihi [20] & 20.03 & $-49.74,89.81$ & 32.84 & $94 \%$ & $<0.00001$ & 0.56 & 0.57 \\
\hline Arevalo [18] & 19.40 & $-51.33,90.13$ & 33.70 & $94 \%$ & $<0.00001$ & 0.54 & 0.59 \\
\hline Jovanović [21] & 21.32 & $-43.01,85.65$ & 40.39 & $95 \%$ & $<0.00001$ & 0.65 & 0.52 \\
\hline
\end{tabular}

$M D$ mean difference, $C I$ confidence interval

IVB alone group and IVB plus MPC group, which indicates that IVB injection is an effective treatment of DME, but the combination of MPC exhibited no additive favorable outcomes.
DME remains a challenging problem that causes severe vision loss in patients with diabetes [22]. The precise pathogenesis has been investigated for decades to find potential 


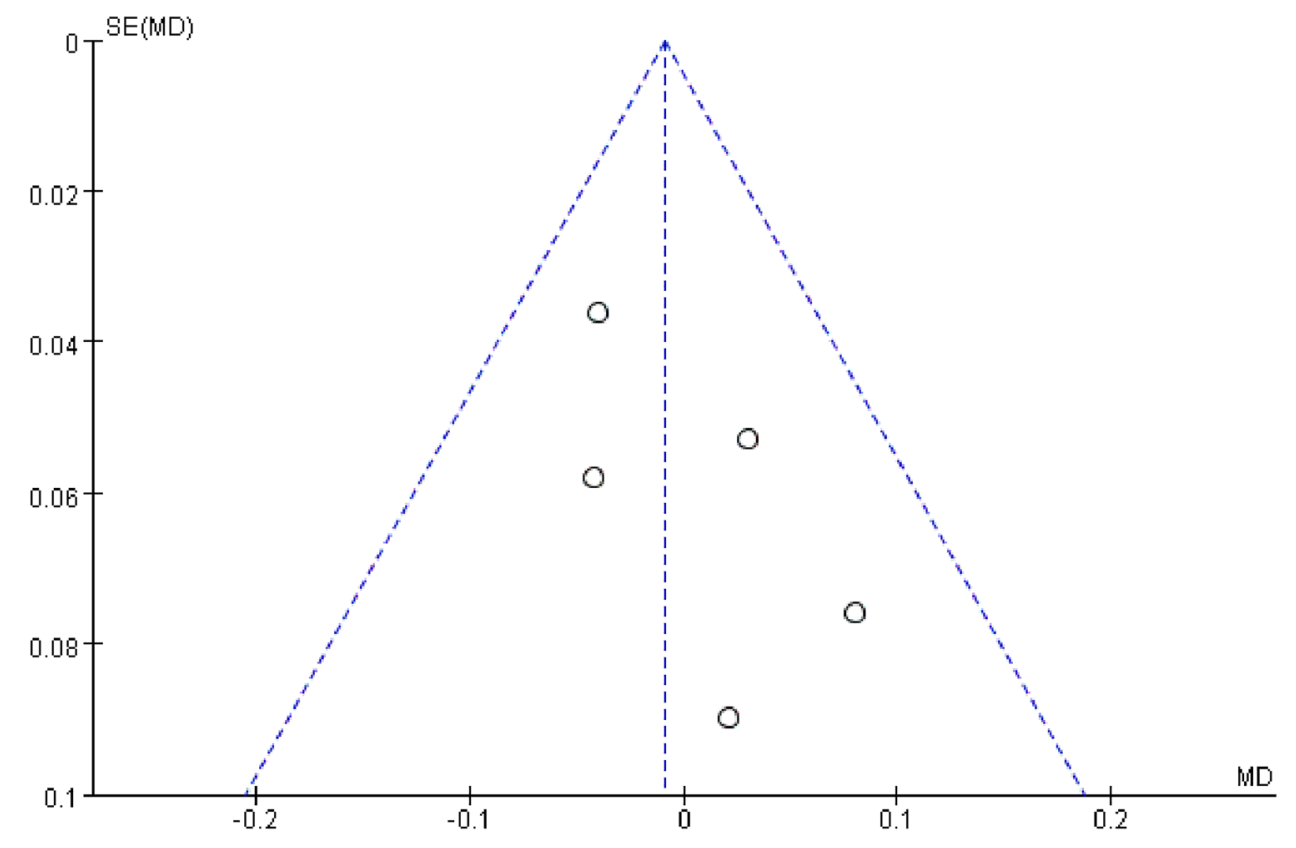

Fig. 5 Funnel plot of publication bias with respect to best-corrected visual acuity (BCVA) at 6 months. $S E$ standard error, $M D$ mean difference

treatment modalities to improve, stabilize, and prevent DME. Because VEGF caused blood-ocular barrier breakdown and hyperpermeability disorder in diabetic retinopathy and macular edema, the anti-VEGF drug bevacizumab is increasingly being used as a therapeutic option for DME [23, 24]. Many studies have reported that IVB injection was effective in reducing DME and improving the BCVA; however, these beneficial effects were transient, and it had no influence on macular hypoxia, which is underlying cause of the problem $[25,26]$. Laser photocoagulation's effect was by a different pathway. It was effective in destroying some photoreceptors to alleviate the high oxygen consumption, and these changes would improve retinal hypoxia [27]. It could be inferred that the combination of MPC with IVB is helpful in reducing macular hypoxia, which may prolong the effect of IVB and produce a better result. Meanwhile, the reduction of macular edema by IVB may render MPC easier and potentiate the effect of MPC. Thus, it is suggested that the combination of MPC with IVB may yield a superior outcome compared with IVB therapy alone.
Both the IVB-alone and combination treatment groups realized a critical reduction of CMT at different follow-up points. We found no significant differences between the two groups regarding CMT at 1, 3, and 6 months after initial treatment. In particular, Solaiman's study showed that the IVB group had a greater reduction in CMT than the combined IVB/MPC group at 1 month, whereas the combined treatment group produced more marked reductions at 3 and 6 months. This was likely due to the transient increase in macular edema after laser photocoagulation in the combined treatment group at 1 month, and the single injection of IVB could not guarantee the effective concentration in the vitreous humor, which caused an increase in the CMT in the IVB group at the longer follow-up periods. In addition, Arevalo's study observed that the IVB group produced a greater decrease in CMT than the combined treatment group throughout the 24 months of follow-up. This was probably because the baseline CMT was higher in the IVB group, which allows more room for improvement to better functional and anatomical outcomes. However, the remaining studies showed no differences in CMT between the two groups. 
In terms of BCVA, we found no significant differences between the IVB-alone and combination treatment groups with regard to BCVA at 1,3 , and 6 months after initial treatment. The improvement in BCVA was not always statistically significant throughout the follow-up. In Solaiman's study, neither group had significant improvement in BCVA at 6 months, which was not parallel to the corresponding CMT changes in the eyes. This could be because many factors could affect the visual outcome, such as the duration, extent, and severity of macular edema, presence of foveal hard exudates, age of the patient, and status of the macula before the onset of edema $[28,29]$. In addition, Lee's study showed that no significant differences existed between the tho groups from 3 months to 6 months, whereas visual acuity in the bevacizumab injection-only group decreased and the combination treatment group showed relatively no change of visual acuity. The results indicate that the combination treatment is beneficial for the maintenance of visual acuity in a longer follow-up period. However, Arevalo's study found the improvement rate of BCVA throughout the 24 months of follow-up was not statistically significantly different between the IVB group and the combination treatment group, which was probably due to the selection bias of patients in the latter group. Seventy-five percent of the patients in the combination treatment group were insulin-dependent, which generally denotes poor glycemic control at some point in therapy and thus influences the therapeutic effect [30]. Additional studies are needed to further assess the effect of MPC in stabilizing visual acuity in the long term.

There was significant heterogeneity among studies evaluating CMT at 1, 3, and 6 months after initial treatment. Sensitivity analysis revealed that the Solaiman study was the source of statistical heterogeneity in the meta-analysis for the CMT at 6 months. There was no evidence of heterogeneity in the three remaining studies after excluding the Solaiman study. Several factors may be attributed to the Solaiman study's heterogeneity. First, the relatively small sample size might have been the main source of heterogeneity. Second, only a single injection of IVB was administered in both groups in this study but not repeated injections, as other studies reported. Third, some factors were not considered in this study, such as degree of glycemic control, level of cholesterol, and other lipids as well as presence of hypertension and renal disease, which might affect macular edema and the treatment outcome [31-33]. Moreover, sensitivity analysis did not alter the result of the previous analysis, which indicates that the combined results were robust and reliable.

Recently, some studies have reported that multiple injections of bevacizumab are beneficial to maintain its transient effect in reducing DME [10, 34], and addition of MPC to the primary IVB therapy helped decrease the number of injections needed for treatment [27]. However, in our study, there was no statistically significant difference between the IVB group and combination treatment group with respect to the number of injections. The unexpected results were probably due to the poor metabolic control in the combination treatment group in Faghihi's and Arevalo's studies and the limited sample size in the combination treatment group in Lee's study. In the future, more studies are needed to verify whether the addition of MPC to IVB therapy could reduce the burden of more frequent injections.

Several limitations of this study should be acknowledged. First, only three RCTs were included, and only one study had adequate sequence generation; none of them had adequate methods to achieve allocation concealment. Moreover, the sample number was limited, which may have influenced the strength of our study. Second, some of the included studies failed to adjust for common confounding variables such as the degree of glycemic control and level of serum lipids, which might have affected the pooled results. Third, the results evaluating CMT were of significant heterogeneity among the studies.

\section{CONCLUSION}

This meta-analysis compared the therapeutic effect of IVB alone to IVB with MPC in DME. The findings suggest that IVB injection is an 
effective modality of treatment in DME, but combination with MPC appeared to have no additive synergistic improvement in CMT and BCVA. However, few studies provide sufficient data about the therapeutic effect of IVB alone and IVB/MPC combination therapy at relatively longer follow-up periods. Given the limitations of this meta-analysis, this conclusion should be interpreted with caution. Meanwhile, further studies are required to elucidate the long-term effects of the two treatment modalities in treating DME.

\section{ACKNOWLEDGEMENTS}

We thank the patients whose data contributed to this study.

Funding. This work was supported by the Science Technological Innovation Outstanding Talents of Henan Province (no. 2018JR0005). The article processing charges were funded by the authors. All authors had full access to all of the data in this study and take complete responsibility for the integrity of the data and accuracy of the data analysis.

Authorship. All named authors meet the International Committee of Medical Journal Editors (ICMJE) criteria for authorship for this article, take responsibility for the integrity of the work as a whole, and have given their approval for this version to be published.

Disclosures. Kang Xiao, Shi-Jia Weng, ShenZhi Liang, Jiong Wang, Cheng Qian, and Guang-Ming Wan have nothing to disclose.

Compliance with Ethics Guidelines. This article is based on previously conducted studies and does not contain any studies with human participants or animals performed by any of the authors.

Data Availability. The data sets generated during and/or analyzed during the current study are available from the corresponding author on reasonable request.
Open Access. This article is distributed under the terms of the Creative Commons Attribution-NonCommercial 4.0 International License (http://creativecommons.org/licenses/ by-nc/4.0/), which permits any noncommercial use, distribution, and reproduction in any medium, provided you give appropriate credit to the original author(s) and the source, provide a link to the Creative Commons license, and indicate if changes were made.

\section{REFERENCES}

1. Zimmet P, Alberti KG, Shaw J. Global and societal implications of the diabetes epidemic. Nature. 2001;414(6865):782-7.

2. Ciulla TA, Amador AG, Zinman B. Diabetic retinopathy and diabetic macular edema: pathophysiology, screening, and novel therapies. Diabetes Care. 2003;26(9):2653-64.

3. Antcliff RJ, Marshall J. The pathogenesis of edema in diabetic maculopathy. Semin Ophthalmol. 1999;14(4):223-32.

4. Photocoagulation for Diabetic Macular Edema. Early treatment diabetic retinopathy study report number 1. Early treatment diabetic retinopathy study research group. Arch Ophthalmol. 1985;103(12):1796-806.

5. Schatz H, Madeira D, McDonald HR, Johnson RN. Progressive enlargement of laser scars following grid laser photocoagulation for diffuse diabetic macular edema. Arch Ophthalmol. 1991;109(11):1549-51.

6. Lee CM, Olk RJ. Modified grid laser photocoagulation for diffuse diabetic macular edema. Long-term visual results. Ophthalmology. 1991;98(10):1594-602.

7. Olk RJ. Modified grid argon (blue-green) laser photocoagulation for diffuse diabetic macular edema. Ophthalmology. 1986;93(7):938-50.

8. Haritoglou C, Kook D, Neubauer A, Wolf A, Priglinger S, Strauss R, et al. Intravitreal bevacizumab (Avastin) therapy for persistent diffuse diabetic macular edema. Retina. 2006;26(9):999-1005.

9. Lam DS, Lai TY, Lee VY, Chan CK, Liu DT, Mohamed S, et al. Efficacy of $1.25 \mathrm{MG}$ versus 2.5 MG intravitreal bevacizumab for diabetic macular edema: six-month results of a randomized controlled trial. Retina. 2009;29(3):292-9. 
10. Arevalo JF, Fromow-Guerra J, Quiroz-Mercado H, Sanchez JG, Wu L, Maia M, et al. Primary intravitreal bevacizumab (Avastin) for diabetic macular edema: results from the Pan-American Collaborative Retina Study Group at 6-month follow-up. Ophthalmology. 2007;114(4):743-50.

11. Seo JW, Park IW. Intravitreal bevacizumab for treatment of diabetic macular edema. Korean J Ophthalmol. 2009;23(1):17-22.

12. Lynch SS, Cheng CM. Bevacizumab for neovascular ocular diseases. Ann Pharmacother. 2007;41(4):614-25.

13. Soheilian M, Ramezani A, Bijanzadeh B, Yaseri M, Ahmadieh $\mathrm{H}$, Dehghan $\mathrm{MH}$, et al. Intravitreal bevacizumab (avastin) injection alone or combined with triamcinolone versus macular photocoagulation as primary treatment of diabetic macular edema. Retina. 2007;27(9):1187-95.

14. Solaiman KA, Diab MM, Abo-Elenin M. Intravitreal bevacizumab and/or macular photocoagulation as a primary treatment for diffuse diabetic macular edema. Retina. 2010;30(10):1638-45.

15. Krohne TU, Eter N, Holz FG, Meyer CH. Intraocular pharmacokinetics of bevacizumab after a single intravitreal injection in humans. Am J Ophthalmol. 2008;146(4):508-12.

16. Higgins JP, Altman DG, Gotzsche PC, Juni P, Moher $\mathrm{D}$, Oxman AD, et al. The Cochrane Collaboration's tool for assessing risk of bias in randomised trials. BMJ. 2011;343:d5928.

17. Stang A. Critical evaluation of the Newcastle-Ottawa Scale for the assessment of the quality of nonrandomized studies in meta-analyses. Eur J Epidemiol. 2010;25(9):603-5.

18. Arevalo JF, Lasave AF, Wu L, Diaz-Llopis M, Gallego-Pinazo R, Alezzandrini AA, et al. Intravitreal bevacizumab plus grid laser photocoagulation or intravitreal bevacizumab or grid laser photocoagulation for diffuse diabetic macular edema: results of the Pan-American Collaborative Retina Study Group at 24 months. Retina. 2013;33(2):403-13.

19. Lee SJ, Kim ET, Moon YS. Intravitreal bevacizumab alone versus combined with macular photocoagulation in diabetic macular edema. Korean J Ophthalmol. 2011;25(5):299-304.

20. Faghihi H, Esfahani MR, Harandi ZA, Madani S. Intravitreal bevacizumab vs. combination of intravitreal bevacizumab plus macular photocoagulation in clinically significant diabetic macular edema: 6 months results of a randomized clinical trial. Iran J Ophthalmol. 2010;22(1):21-6.
21. Javanovic S, Canadanovic V, Sabo A, Grgic Z, Mitrovic M, Rakic D. Intravitreal bevacizumab injection alone or combined with macular photocoagulation compared to macular photocoagulation as primary treatment of diabetic macular edema. Vojnosanit Pregl. 2015;72(10):876-82.

22. Romero-Aroca P. Current status in diabetic macular edema treatments. World J Diabetes. 2013;4(5):165-9.

23. Fortin $\mathrm{P}$, Mintzes B, Innes M. A systematic review of intravitreal bevacizumab for the treatment of diabetic macular edema. CADTH Technol Overv. 2013;3(1):e3203.

24. Forte R, Cennamo G, Breve MA, Vecchio EC, de Crecchio G. Functional and anatomic response of the retina and the choroid to intravitreal bevacizumab for macular edema. J Ocul Pharmacol Ther. 2012;28(1):69-75.

25. Kook D, Wolf A, Kreutzer T, Neubauer A, Strauss R, Ulbig $M$, et al. Long-term effect of intravitreal bevacizumab (avastin) in patients with chronic diffuse diabetic macular edema. Retina. 2008;28(8):1053-60.

26. Shimura M, Nakazawa T, Yasuda K, Shiono T, Iida T, Sakamoto T, et al. Comparative therapy evaluation of intravitreal bevacizumab and triamcinolone acetonide on persistent diffuse diabetic macular edema. Am J Ophthalmol. 2008;145(5):854-61.

27. Solaiman KA, Diab MM, Dabour SA. Repeated intravitreal bevacizumab injection with and without macular grid photocoagulation for treatment of diffuse diabetic macular edema. Retina. 2013;33(8):1623-9.

28. Eriksson U, Alm A, Bjarnhall G, Granstam E, Matsson AW. Macular edema and visual outcome following cataract surgery in patients with diabetic retinopathy and controls. Graefes Arch Clin Exp Ophthalmol. 2011;249(3):349-59.

29. Otani T, Kishi S. Tomographic findings of foveal hard exudates in diabetic macular edema. Am J Ophthalmol. 2001;131(1):50-4.

30. Macky TA, Mahgoub MM. The effect of glycemic control on visual and anatomic outcomes in response to therapy for diabetic macular edema. Eur J Ophthalmol. 2012;23:100.

31. Matsuda S, Tam T, Singh RP, Kaiser PK, Petkovsek $D$, Carneiro $G$, et al. The impact of metabolic parameters on clinical response to VEGF inhibitors for diabetic macular edema. J Diabetes Complic. 2014;28(2):166-70. 
32. Ucgun NI, Yildirim Z, Kilic N, Gursel E. The importance of serum lipids in exudative diabetic macular edema in type 2 diabetic patients. Ann N Y Acad Sci. 2007;1100:213-7.

33. Lopes de Faria JM, Jalkh AE, Trempe CL, McMeel JW. Diabetic macular edema: risk factors and concomitants. Acta Ophthalmol Scand. 1999;77(2): $170-5$.
34. Lee CM, Olk RJ, Akduman L. Combined modified grid and panretinal photocoagulation for diffuse diabetic macular edema and proliferative diabetic retinopathy. Ophthalmic Surg Lasers. 2000;31(4): 292-300. 